\title{
Article \\ Reconfigurable Intelligent Surface in Wireless-Powered Interference-Limited Communication Networks
}

\author{
Kehinde Odeyemi ${ }^{1,2, *}$, Pius Owolawi ${ }^{1}$ (1) and Oladayo Olakanmi ${ }^{2}$ \\ 1 Department of Computer Systems Engineering, Tshwane University of Technology, \\ Pretoria 0001, South Africa; p.owolawi@gmail.com or owolawipa@tut.ac.za \\ 2 Department of Electrical and Electronic Engineering, Faculty of Technology, University of Ibadan, \\ Ibadan 200284, Nigeria; olarad4@yahoo.com or olakanmi.oladayo@ui.edu.ng \\ * Correspondence: ko.odeyemi@ui.edu.ng
}

check for updates

Citation: Odeyemi, K.; Owolawi, P.; Olakanmi, O. Reconfigurable Intelligent Surface in Wireless-Powered Interference-Limited Communication Networks. Symmetry 2021, 13, 960 https://doi.org/10.3390/ sym 13060960

Academic Editor: Jan Awrejcewicz

Received: 12 April 2021

Accepted: 7 May 2021

Published: 28 May 2021

Publisher's Note: MDPI stays neutral with regard to jurisdictional claims in published maps and institutional affiliations.

Copyright: (c) 2021 by the authors. Licensee MDPI, Basel, Switzerland. This article is an open access article distributed under the terms and conditions of the Creative Commons Attribution (CC BY) license (https:// creativecommons.org/licenses/by/ $4.0 /)$.

\begin{abstract}
The commercialization of future wireless communication systems faces crucial problems in terms of reduced network cost of deployment and high power consumption. As a result, reconfigurable intelligent surfaces (RIS) have been suggested as a promising approach to overcome these existing challenges. In this paper, the performance of RIS in a wireless-powered interference-limited communication network is investigated. In this network, an energy-constrained access point (AP) is powered by a dedicated multiantenna power beacon (PB) and communicates to a destination limited-interference node via the RIS. It is assumed that the RIS experiences generalized-K fading distribution while the PB and interferers links are subjected to Nakagami-m fading distributions. To evaluate the system performance, the analytical closed-form expression of the probability distribution function (PDF) for the concerned system is derived. Through this, the exact closed-form expressions of the systems outage probability, average delay-tolerance throughput and average bit error rate are obtained. The analysis quantifies the effects of the number of reflecting elements in the RIS, number of interferer nodes, fading parameters on the AP and interferer links and number of antennas on the PB. It is deduced from the results that there is a kind of symmetry relationship between the analytical and simulation results. Additionally, the results illustrate that the proposed system with an RIS outperforms the conventional system without an RIS. Finally, the accuracy of the derived analytical expressions is validated through a Monte-Carlo simulation.
\end{abstract}

Keywords: energy harvesting; interferer nodes; power beacon; reconfigurable intelligent surface

\section{Introduction}

\subsection{Background Information}

Today, reconfigurable intelligent surfaces (RIS) have emerged as a new innovative technology in the research community due to its great potential for reconfiguring propagation environments and enhancing the quality of signal reception [1]. RIS is expected to play a key role in improving the coverage area, energy efficiency and data rates of future wireless networks such as fifth-generation (5G) and beyond [2]. Compared with conventional systems, it requires no additional power supply, complex encoding or decoding operation to enhance the system's performance $[3,4]$. In this case, the RIS concept is based on the utilization of large number of small, low-cost and passive reflecting elements to effectively control the propagation characteristics of the desired incident signal through the adjustable amplitude and phase shift of each reflecting element with no signal processing [4]. Practically, an RIS can be fabricated to conform to any infrastructural surfaces such as walls, ceiling and buildings for easy signal reflection [5]. As a result of this, an RIS eliminates the blockage and fading effects that are usually experienced by large-scale antenna wireless systems due to the existence of trees, buildings and humans [3], and also reduces the system's complexity and cost [6]. 
Recently, wireless energy harvesting $(\mathrm{EH})$ has been suggested as one promising technique to prolong the lifetime of energy-constrained wireless devices [7]. It involves the conversion of received RF energy to electrical power to boost the power of wireless devices. Generally, EH can be classified as wireless-powered communication networks (WPCNs) and simultaneous wireless information and power transfer (SWIPT). The formal technique involves the AP scavenging RF energy from a dedicated PB and employing the energy to send its information to the system destination. The latter technique usually employs energy-constrained relay nodes to harvest RF energy from the AP and uses the energy to transmit information to the system destination $[8,9]$. In both cases, there are three EH protocols, and this includes the time switching (TS) protocol, power splitting (PS), and ideal relaying protocol which are detailed in $[10,11]$. Thus, the performance of both approaches has been studied in literature in the context of cooperative relaying systems.

\subsection{Related Works}

Recently, various research works have evaluated the performance of RIS in wireless communication systems. Specifically, the performance of a RIS-assisted UAV relaying system was investigated in [12]. Additionally, the performance of an RIS-aided power line communication system was studied in [13] where the RIS was employed to improve system coverage. The authors in [4] evaluated the performance of an RIS-enhanced underwater communication system. The performance of an RIS-aided network over a Nakagami$\mathrm{m}$ fading channel was investigated in [6]. In [14], the performance of an RIS-assisted source multi-user mixed radio frequency/free space optical (FSO) relay network was evaluated with opportunistic user scheduling. Moreover, the performance of the RISassisted mobile network under random user mobility was investigated in [15]. The authors in [16] presented the performance of an RIS-enhanced internet of things network over an F-distribution fading channel. Further, the impact of modulation index techniques on an RIS-based space shift keying wireless network was evaluated in [17]. In [18], the performance analysis of an RIS-assisted non-orthogonal multiple access network was presented. Yang et al. [19] considered RIS in an FSO setup under the influence of atmospheric turbulence and pointing error. The authors in $[20,21]$ studied the security performance in vehicle networks with different modes of RIS. However, in all the aforementioned works, the systems under study did not considered co-channel interference. As a matter of this, in [22], the performance of RIS in an interference-limited RIS-aided network was studied, where the interference links at the system destination were subjected to Rayleigh fading distribution. It is worth noting that the system model in [22] did not consider a wireless-powered communication technique. To the best of authors' knowledge, the RIS in interference-limited networks in context of a wireless-powered communication scenario has not been yet studied in literature.

\subsection{Motivation and Contributions}

Motivated by this, the performance of RIS in a wireless-powered interference limited communication system is presented in this paper. In this system, an energy-constrained AP harvests energy from a dedicated multi-antenna $\mathrm{PB}$ and uses the energy to send its information to a destination limited-interference node via the RIS. The RIS link is subjected to generalised- $K$ fading distribution while the $P B$ and interferer links experience Nakagami- $m$ fading distributions. To this end, the system end-to-end probability distribution function is obtained, through which the exact closed-form expressions of the system outage probability, average delay-tolerance throughput and average bit error rate are obtained. Additionally, a kind of symmetry can be deduced in the relationship between the analytical results and the simulated results, which proves the accuracy of the derived analytical expression. The main contributions of this study are summarised as follows:

- A closed-form expression of the system end-to-end probability distribution function is derived,

- The exact closed-form expression for the system outage probability is obtained, 
- The analytical expression of the system average throughput under the delay-tolerant transmission mode is also derived,

- The exact closed-form expression for the system average bit error rate is obtained under the binary and coherent modulation scheme, and

- Related to [22], the co-channel interference system considered in this work utilizes wireless-powered communication technique.

\subsection{Structure of the Paper}

The reminder of this paper is structured as follows: in Section 2, the system and channel models are presented. The system statistical characteristics are detailed in Section 3. In Section 4, the system performance metrics are analysed. The numerical results and discussions are illustrated in Section 5. Finally, Section 6 depicts the concluding remarks of this work.

\section{System and Channel Models}

A wireless powered interference-limited communication network that consists of a power beacon (PB), access point (AP), RIS, a destination (D) and multiple $N_{I}$ interferer nodes is illustrated in Figure 1. The PB and RIS are equipped with $N_{P}$ antennas and $N$ reflecting elements respectively while the $\mathrm{AP}$, destination and interferer nodes are equipped with a single antenna. Owing to blockage and fading issues, it is assumed that there is no direct link between the AP and the destination. In the network, the AP harvests RF energy from the PB using a time-splitting protocol and uses the energy to transmit its information to the destination via the RIS. Based on time-splitting, the overall transmission time $T$ is divided into two phases. During the first transmission phase, the AP harvests RF energy from the PB with duration $\rho T$ and the $\rho(0<\rho<1)$ is the time-splitting ratio. During the second transmission phase with duration $(1-\rho) T$, the AP utilizes the harvested RF energy to transmit its information to the destination. Thus, the signal received at the AP can then be expressed as:

$$
y_{A P}=\sqrt{P_{s} d_{p}^{-\zeta}} h_{p} x_{p}+z_{p}
$$

where $P_{S}$ denotes the PB transmit power, $d_{p}$ is the distance between the PB and AP with $\zeta$ signifying the path loss-exponent, $h_{p}$ is the channel gain between the $\mathrm{PB}$ and $\mathrm{AP}, x_{p}$ is the unit energy signal and $z_{p}$ denotes the additive white Gaussian noise (AWGN) with noise variance $\sigma_{p}^{2}$ and zero mean. Moreover, the amount of energy harvested by the AP at the energy period $\rho T$ can be thus expressed as:

$$
E_{p}=\frac{\beta \rho T P_{s}}{d_{p}^{\zeta}},
$$

where $\beta$ signifies the energy conversion efficiency at $0<\beta<1$.

Therefore, the transmit power at the AP during the information transmission period $(1-\rho) T$ can be expressed as:

$$
P_{t}=\frac{E_{p}}{(1-\rho) T} \triangleq \frac{\beta \rho P_{s}}{(1-\rho) d_{p}^{\zeta}},
$$

At the system destination, the received signal can then be given as:

$$
y_{D}=\sqrt{P_{t} D_{R}^{-\zeta}}\left[\sum_{i=1}^{N} \alpha_{i} \exp ^{j \phi_{i}} \eta_{i}\right] x_{t}+\sum_{j=1}^{N_{I}} \sqrt{P_{I_{j}} d_{I_{j}}^{-\zeta}} h_{I_{j}} x_{I_{j}},
$$




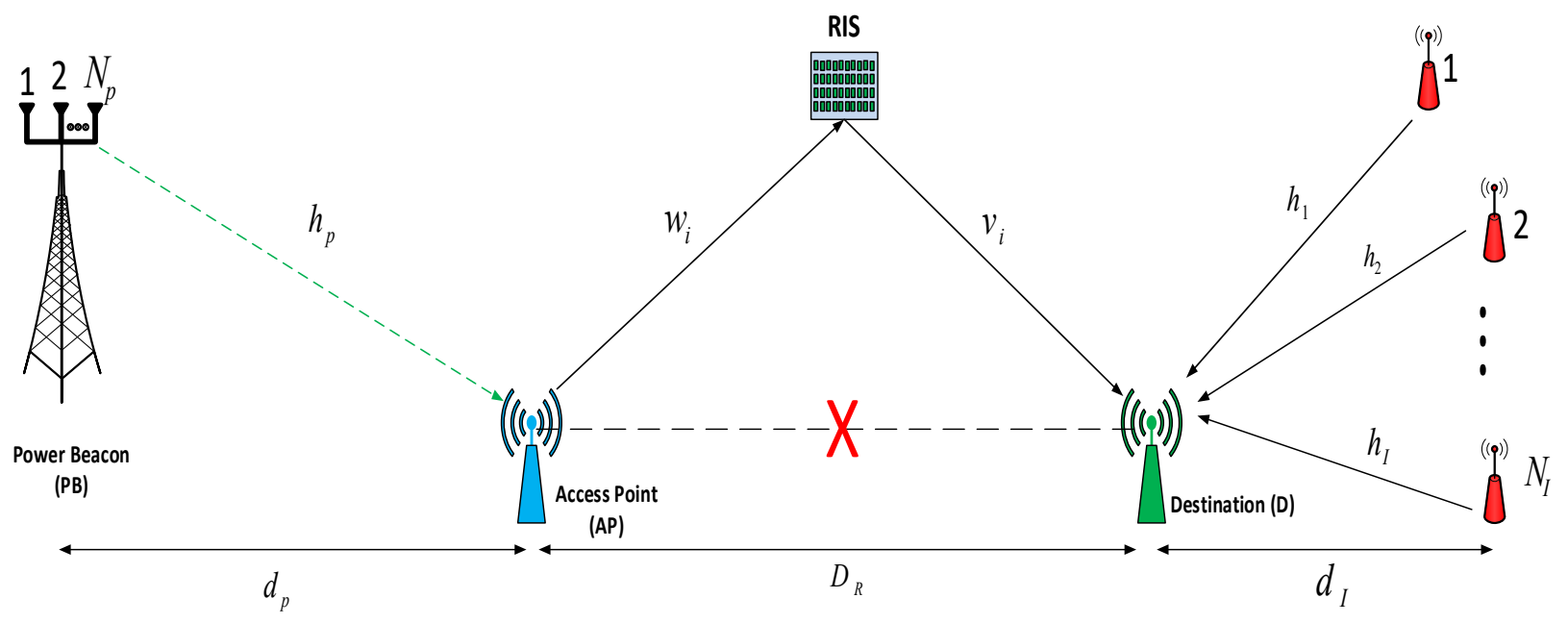

Figure 1. System model for an RIS aided interference-limited wireless powered network.

where $x_{t}$ is the transmit information, $\phi_{i}$ denotes the phase shift produced by $i$-th reflecting elements of the RIS, $\alpha_{i}=\omega_{i} \exp ^{-\psi_{i}} / \sqrt{L_{A R}}$ and $\eta_{i}=v_{i} \exp ^{-\vartheta_{i}} / \sqrt{L_{R D}}$ are the channel gain of RIS with $\omega_{i}$ and $v_{i}$ are the Rayleigh and Rician random variable, respectively. $L_{A R}=10 \log 10\left(d_{A R}^{\zeta}\right)+\lambda$ and $L_{R D}=10 \log 10\left(d_{R D}^{\zeta}\right)+\lambda$ are the RIS link path loss where $d_{A R}$ and $d_{R D}$ are, respectively, the distances between the AP-to-RIS and RIS-to-D links, and $\lambda$ denotes the constant that depends on the signal frequency and transmission environment. Moreover, the channel gain and the transmit power of the $j$-th interferer are denoted respected as $h_{I_{j}}$ and $P_{I_{j}}$. Additionally, $d_{I_{j}}$ signifies the distance between the destination and the $j$-th interferer.

The maximum SNR for the system can be obtained by setting the $\phi_{i}=\psi_{i}+\vartheta_{i}$, then the (4) can be further expressed as:

$$
y_{D}=\sqrt{P_{t} D_{R}^{-\zeta}}\left[\sum_{i=1}^{N} \omega_{i} v_{i}\right] x_{t}+\sum_{j=1}^{N_{I}} \sqrt{P_{I_{j}} d_{I_{j}}^{-\zeta}} h_{I_{j}} x_{I_{j}},
$$

Through (1), (3) and (5), the received signal-to-interference ratio (SIR) at the destination can be expressed as:

$$
\gamma=\xi\left\|h_{P}\right\|^{2} \frac{\left\|h_{R}\right\|^{2}}{\left\|h_{I_{j}}\right\|^{2}}
$$

where $\xi=\frac{\beta \rho P_{s}}{(1-\rho) \sigma^{2} d_{P}^{\zeta} D_{R}^{\zeta} P_{I} d_{I}^{-\zeta}}$ with $D_{R}=L_{A R} L_{R D}$ and $h_{R}=\sum_{i=1}^{N} \omega_{i} v_{i}$.

In this paper, it is assumed that the RIS link is subjected to an approximated generalised K-distribution and the PDF of the sum of multiple generalised $\mathrm{K}$ random variables of $h_{R}$ can be defined as [23]:

$$
f_{h_{R}}(y)=\frac{2 \Psi^{k_{q}+m_{q}} y^{\left(\left(k_{q}+m_{q}\right) / 2-1\right)}}{\Gamma\left(k_{q}\right) \Gamma\left(m_{q}\right)\left(\bar{\gamma}_{R} / D_{R}\right)^{\left(k_{q}+m_{q}\right) / 2}} K_{k_{q}-m_{q}}\left(2 \Psi \sqrt{\frac{y D_{R}}{\bar{\gamma}_{R}}}\right)
$$

where $k_{q}$ and $m_{q}$ represent the distribution parameters and $\Psi=\sqrt{k_{q} m_{q} / \Omega_{q}}$ with $\Omega_{q}$ denoting the mean power. $K_{v}($.$) signifies the modified Bessel function of the second kind$ with zero order and $\Gamma($.$) is the Gamma function. \bar{\gamma}_{R}$ is the average SNR of the RIS link.

By converting the PDF to a Meijer-G function using the identity stated in [24], Equation (14), the (7) can be further expressed as: 


$$
\begin{aligned}
& f_{Y}(y)=\frac{\Psi^{k_{q}+m_{q}} y^{\left(\left(k_{q}+m_{q}\right) / 2-1\right)}}{\Gamma\left(k_{q}\right) \Gamma\left(m_{q}\right)\left(\bar{\gamma}_{R} / D_{R}\right)^{\left(k_{q}+m_{q}\right) / 2}} G_{0,2}^{2,0}\left(\frac{\Psi^{2} D_{R}}{\bar{\gamma}_{R}} y \mid\left(k_{q}+m_{q}\right) / 2, \quad\left(m_{q}-k_{q}\right) / 2\right) . \\
& f_{h_{w}}^{w}(x)=\left(\frac{m_{w}}{\bar{\gamma}_{w}}\right)^{m_{w} N_{w}} \frac{x^{m_{w} N_{w}-1}}{\Gamma\left(m_{w} N_{w}\right)} \exp \left(-\frac{m_{w}}{\bar{\gamma}_{w}} x\right) \\
& f_{Y}(y)=\int_{0}^{\infty} x f_{h_{R}}(y x) f_{h_{I}}^{I}(x) d x \\
& f_{Y}(y)=\frac{\Psi^{k_{q}+m_{q}} y^{\left(\left(k_{q}+m_{q}\right) / 2-1\right)}}{\Gamma\left(k_{q}\right) \Gamma\left(m_{q}\right) \Gamma\left(m_{I} N_{I}\right)\left(\bar{\gamma}_{R} / D_{R}\right)^{\left(k_{q}+m_{q}\right) / 2}}\left(\frac{m_{I}}{\bar{\gamma}_{I}}\right)^{m_{I} N_{I}}
\end{aligned}
$$

\section{System Statistical Characteristics}

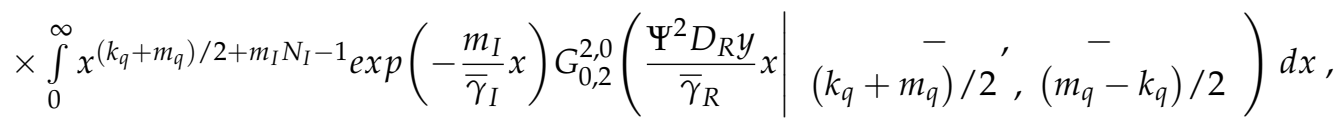

By applying the integral identity defined in ([27], Equation (7.813(1))), (11) can be solved as:

$$
f_{Y}(y)=\frac{\Psi^{k_{q}+m_{q}} y^{\left(\left(k_{q}+m_{q}\right) / 2-1\right)}}{\Gamma\left(k_{q}\right) \Gamma\left(m_{q}\right) \Gamma\left(m_{I} N_{I}\right)\left(\bar{\gamma}_{R} / D_{R}\right)^{\left(k_{q}+m_{q}\right) / 2}}\left(\frac{m_{I}}{\bar{\gamma}_{I}}\right)^{-\left(k_{q}+m_{q}\right) / 2} G_{1,2}^{2,1}\left(\frac{\Psi^{2} \bar{\gamma}_{I} D_{R}}{m_{I} \bar{\gamma}_{R}} y \mid \begin{array}{c}
1-\left(k_{q}+m_{q}\right) / 2-m_{I} N_{I} \\
\left(k_{q}+m_{q}\right) / 2,\left(m_{q}-k_{q}\right) / 2
\end{array}\right)
$$

Thereafter, the PDF of the product of two random variables such that $Z=Y .\left\|h_{p}\right\|^{2}$ can be obtained as:

$$
f_{Z}(z)=\int_{0}^{\infty} \frac{1}{x} f_{Y}\left(\frac{z}{x}\right) f_{h_{p}}^{P}(x) d x
$$

Also, by invoking (11) and (9) into (13), (13) can be expressed as:

$$
\begin{gathered}
f_{Z}(z)=\frac{\Psi^{k_{q}+m_{q}}{ }^{\left(\frac{\left(k_{q}+m_{q}\right)}{2}-1\right)}}{\Gamma\left(k_{q}\right) \Gamma\left(m_{q}\right) \Gamma\left(m_{I} N_{I}\right) \Gamma\left(m_{P} N_{P}\right)\left(\frac{\bar{\gamma}_{R}}{D_{R}}\right)^{\frac{\left(k_{q}+m_{q}\right)}{2}}\left(\frac{m_{I}}{\bar{\gamma}_{I}}\right)^{-\frac{\left(k_{q}+m_{q}\right)}{2}}\left(\frac{m_{P}}{\bar{\gamma}_{P}}\right)^{m_{P} N_{P}}} \\
\times \int_{0}^{\infty} x^{m_{P} N_{P}-\left(\left(k_{q}+m_{q}\right) / 2\right)-1} \exp \left(-\frac{m_{p}}{\bar{\gamma}_{p}} x\right) G_{1,2}^{2,1}\left(\frac{\Psi^{2} \bar{\gamma}_{I} D_{R} Z}{m_{I} \bar{\gamma}_{R} x} \mid \begin{array}{c}
1-\left(k_{q}+m_{q}\right) / 2-m_{I} N_{I} \\
\left(k_{q}+m_{q}\right) / 2,\left(m_{q}-k_{q}\right) / 2
\end{array}\right) d x
\end{gathered}
$$

By inverting the Meijer-function in (14) using the identity in ([27], Equation (7.813(1))), then (14) can be further expressed as: 


$$
\begin{gathered}
f_{Z}(z)=\frac{\Psi^{k_{q}+m_{q}} z^{\left(\left(k_{q}+m_{q}\right) / 2-1\right)}}{\Gamma\left(k_{q}\right) \Gamma\left(m_{q}\right) \Gamma\left(m_{I} N_{I}\right) \Gamma\left(m_{P} N_{P}\right)\left(\bar{\gamma}_{R} / D_{R}\right)^{\left(k_{q}+m_{q}\right) / 2}}\left(\frac{m_{I}}{\bar{\gamma}_{I}}\right)^{-\left(k_{q}+m_{q}\right) / 2}\left(\frac{m_{P}}{\bar{\gamma}_{P}}\right)^{m_{P} N_{P}} \\
\times \int_{0}^{\infty} x^{m_{P} N_{P}-\left(\left(k_{q}+m_{q}\right) / 2\right)-1} \exp \left(-\frac{m_{p}}{\bar{\gamma}_{p}} x\right) G_{2,1}^{1,2}\left(\frac{m_{I} \bar{\gamma}_{R} x}{\Psi^{2} \bar{\gamma}_{I} D_{R} Z} \mid \begin{array}{c}
1-\left(k_{q}+m_{q}\right) / 2,1-\left(m_{q}-k_{q}\right) / 2 \\
\left(k_{q}+m_{q}\right) / 2+m_{I} N_{I}
\end{array}\right) d x,
\end{gathered}
$$

By utilizing the integral identity detailed in ([27], Equation (7.813(1))), (15) can be solved as:

$$
\begin{aligned}
& f_{Z}(z)=\frac{\Psi^{k_{q}+m_{q}}}{\Gamma\left(k_{q}\right) \Gamma\left(m_{q}\right) \Gamma\left(m_{I} N_{I}\right) \Gamma\left(m_{P} N_{P}\right)\left(\bar{\gamma}_{R} / D_{R}\right)^{\left(k_{q}+m_{q}\right) / 2}}\left(\frac{m_{P} \bar{\gamma}_{I}}{m_{I} \bar{\gamma}_{P}}\right)^{\left(k_{q}+m_{q}\right) / 2} z^{\left(\left(k_{q}+m_{q}\right) / 2-1\right)} \\
& \times G_{3,1}^{1,3}\left(\frac{m_{I} \bar{\gamma}_{R} \bar{\gamma}_{P}}{\Psi^{2} \bar{\gamma}_{I} m_{P} D_{R} Z} \mid \begin{array}{c}
1+\left(\left(k_{q}+m_{q}\right) / 2\right)-m_{P} N_{P}, 1-\left(k_{q}+m_{q}\right) / 2,1-\left(m_{q}-k_{q}\right) / 2 \\
\left(k_{q}+m_{q}\right) / 2+m_{I} N_{I}
\end{array}\right),
\end{aligned}
$$

By inverting the Meijer-G function in (16) using the identity detailed in ([27], Equation (9.31(5))), (16) can be further expressed as:

$$
\begin{aligned}
f_{Z}(z) & =\frac{\Psi^{k_{q}+m_{q}}}{\Gamma\left(k_{q}\right) \Gamma\left(m_{q}\right) \Gamma\left(m_{I} N_{I}\right) \Gamma\left(m_{P} N_{P}\right)\left(\bar{\gamma}_{R} / D_{R}\right)^{\left(k_{q}+m_{q}\right) / 2}}\left(\frac{m_{P} \bar{\gamma}_{I}}{m_{I} \bar{\gamma}_{P}}\right)^{\left(k_{q}+m_{q}\right) / 2} z^{\left(\left(k_{q}+m_{q}\right) / 2-1\right)} \\
& \times G_{1,3}^{3,1}\left(\frac{\Psi^{2} \bar{\gamma}_{I} m_{P} D_{R} Z}{m_{I} \bar{\gamma}_{R} \bar{\gamma}_{P}} \mid \begin{array}{c}
\left(k_{q}+m_{q}\right) / 2+m_{I} N_{I} \\
m_{P} N_{P}-\left(\left(k_{q}+m_{q}\right) / 2\right),\left(k_{q}+m_{q}\right) / 2,\left(m_{q}-k_{q}\right) / 2
\end{array}\right),
\end{aligned}
$$

Through (6) and transformation of random variable, (17) can be expressed as:

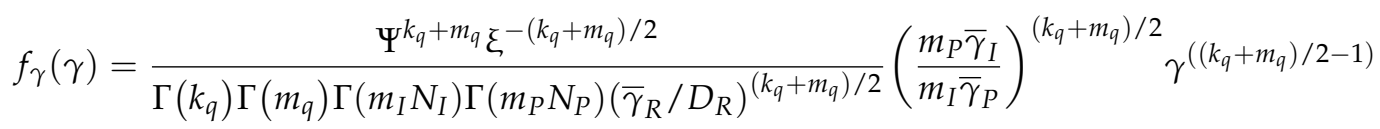

$$
\begin{aligned}
& \times G_{1,3}^{3,1}\left(\frac{\Psi^{2} \bar{\gamma}_{I} m_{P} D_{R} \gamma}{m_{I} \bar{\gamma}_{R} \bar{\gamma}_{P} \xi} \mid \begin{array}{c}
\left(k_{q}+m_{q}\right) / 2+m_{I} N_{I} \\
m_{P} N_{P}-\left(\left(k_{q}+m_{q}\right) / 2\right),\left(k_{q}+m_{q}\right) / 2,\left(m_{q}-k_{q}\right) / 2
\end{array}\right) .
\end{aligned}
$$

\section{System Performance Analysis}

To analyse the performance of the concerned system, the closed-form expressions of the outage probability, average throughput under the delay-tolerant transmission mode and the average BER for binary and coherent modulation are derived in this section.

\subsection{Outage Probability Analysis}

Outage probability is one of the key performance metrics for quantifying the performance of a wireless communication system under the effect of fading. It is defined as the probability that the system's instantaneous SNR falls below the preset threshold value $\gamma_{t h}$. It can thus be obtained as [28]:

$$
P_{\text {out }}\left(\gamma_{t h}\right)=\int_{0}^{\gamma_{t h}} f_{\gamma}\left(\gamma_{t h}\right) d \gamma,
$$

where $\gamma_{t h}=2^{R}-1$ and $R$ denotes the fixed rate.

Substituting (18) into (19) and applying the integral identity detailed in [24], Equation (26), the outage probability of the system can be expressed as:

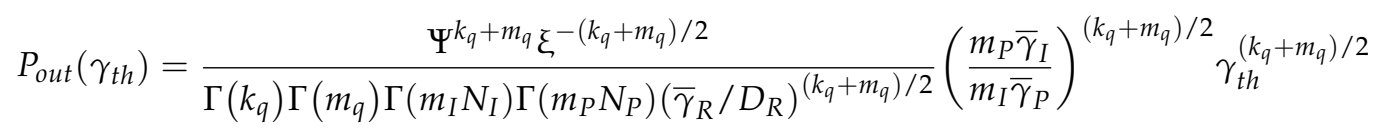

$$
\begin{aligned}
& \times G_{2,4}^{3,2}\left(\frac{\Psi^{2} \bar{\gamma}_{I} m_{P} D_{R} \gamma_{t h}}{m_{I} \bar{\gamma}_{R} \bar{\gamma}_{P} \xi} \mid \begin{array}{c}
\left(k_{q}+m_{q}\right) / 2+m_{I} N_{I}, 1-\left(k_{q}+m_{q}\right) / 2 \\
m_{P} N_{P}-\left(\left(k_{q}+m_{q}\right) / 2\right),\left(k_{q}+m_{q}\right) / 2,\left(m_{q}-k_{q}\right) / 2,-\left(k_{q}+m_{q}\right) / 2
\end{array}\right) .
\end{aligned}
$$




\subsection{Average Throughput Analysis}

The average throughput under the delay-tolerant transmission mode can be obtained by determining the ergodic capacity at any fixed target rate as follows [7]:

$$
\tau=(1-\rho) C,
$$

where $C$ is the ergodic capacity of the system which can be defined as [15,29]:

$$
C=\frac{1}{\ln (2)} \int_{0}^{\infty} \ln (1+\gamma) f_{\gamma}(\gamma) d \gamma,
$$

By substituting (18) into (22), (22) can be expressed as:

$$
\begin{gathered}
C=\frac{\Psi^{k_{q}+m_{q}} \xi^{-\left(k_{q}+m_{q}\right) / 2}\left(\frac{m_{P} \bar{\gamma}_{I}}{m_{I} \bar{\gamma}_{P}}\right)^{\left(k_{q}+m_{q}\right) / 2}}{\ln (2) \Gamma\left(k_{q}\right) \Gamma\left(m_{q}\right) \Gamma\left(m_{I} N_{I}\right) \Gamma\left(m_{P} N_{P}\right)\left(\bar{\gamma}_{R} / D_{R}\right)^{\left(k_{q}+m_{q}\right) / 2}} \int_{0}^{\infty} \gamma^{\left(\left(k_{q}+m_{q}\right) / 2-1\right)} \\
\times \ln (1+\gamma) G_{1,3}^{3,1}\left(\frac{\Psi^{2} \bar{\gamma}_{I} m_{P} D_{R} \gamma}{m_{I} \bar{\gamma}_{R} \bar{\gamma}_{P} \xi} \mid \begin{array}{c}
\left(k_{q}+m_{q}\right) / 2+m_{I} N_{I} \\
m_{P} N_{P}-\left(\left(k_{q}+m_{q}\right) / 2\right),\left(k_{q}+m_{q}\right) / 2,\left(m_{q}-k_{q}\right) / 2
\end{array}\right) d \gamma,
\end{gathered}
$$

By converting $\ln (1+\gamma)$ to the Meijer-G function through the identity stated in [30], Equation (8.4.6(5)), then (23) can be further expressed as:

$$
\begin{gathered}
C=\frac{\Psi^{k_{q}+m_{q}} \xi^{-\left(k_{q}+m_{q}\right) / 2}\left(\frac{m_{P} \bar{\gamma}_{I}}{m_{I} \bar{\gamma}_{P}}\right)^{\left(k_{q}+m_{q}\right) / 2}}{\ln (2) \Gamma\left(k_{q}\right) \Gamma\left(m_{q}\right) \Gamma\left(m_{I} N_{I}\right) \Gamma\left(m_{P} N_{P}\right)\left(\bar{\gamma}_{R} / D_{R}\right)^{\left(k_{q}+m_{q}\right) / 2}} \int_{0}^{\infty} \gamma^{\left(\left(k_{q}+m_{q}\right) / 2-1\right)} \\
\times G_{1,3}^{3,1}\left(\begin{array}{c}
1,1 \\
1,0
\end{array}\right) G_{1,3}^{3,1}\left(\frac{\Psi^{2} \bar{\gamma}_{I} m_{P} D_{R} \gamma}{m_{I} \bar{\gamma}_{R} \bar{\gamma}_{P} \xi} \mid \begin{array}{c}
\left(k_{q}+m_{q}\right) / 2+m_{I} N_{I} \\
m_{P} N_{P}-\left(\left(k_{q}+m_{q}\right) / 2\right),\left(k_{q}+m_{q}\right) / 2,\left(m_{q}-k_{q}\right) / 2
\end{array}\right) d \gamma,
\end{gathered}
$$

By applying the integral identity detailed in [24], Equations (21) and (24) can be expressed as:

$$
\begin{gathered}
C=\frac{\Psi^{k_{q}+m_{q}} \xi^{-\left(k_{q}+m_{q}\right) / 2}}{\ln (2) \Gamma\left(k_{q}\right) \Gamma\left(m_{q}\right) \Gamma\left(m_{I} N_{I}\right) \Gamma\left(m_{P} N_{P}\right)\left(\bar{\gamma}_{R} / D_{R}\right)^{\left(k_{q}+m_{q}\right) / 2}}\left(\frac{m_{P} \bar{\gamma}_{I}}{m_{I} \bar{\gamma}_{P}}\right)^{\left(k_{q}+m_{q}\right) / 2} \\
\times G_{3,5}^{5,5}\left(\frac{\Psi^{2} \bar{\gamma}_{I} m_{P} D_{R} \gamma}{m_{I} \bar{\gamma}_{R} \bar{\gamma}_{P} \xi} \mid \begin{array}{c}
\frac{\left(k_{q}+m_{q}\right)}{2}+m_{I} N_{I},-\frac{\left(k_{q}+m_{q}\right)}{2}, 1-\frac{\left(k_{q}+m_{q}\right)}{2} \\
m_{P} N_{P}-\frac{\left(k_{q}+m_{q}\right)}{2}, \frac{\left(k_{q}+m_{q}\right)}{2}, \frac{\left(m_{q}-k_{q}\right)}{2},-\frac{\left(k_{q}+m_{q}\right)}{2},-\frac{\left(k_{q}+m_{q}\right)}{2}
\end{array}\right) .
\end{gathered}
$$

Thus, the average throughput can then be obtained by putting (25) into (21).

\subsection{Average Bit Error Rate Analysis}

\subsubsection{For Binary Modulation}

The average BER for several binary modulation schemes can be expressed as [7]:

$$
P_{b}=\frac{1}{2 \Gamma(\psi)} \int_{0}^{\infty} f_{\gamma}(\gamma) \Gamma(\psi, \mu x) d \gamma,
$$

where $\psi$ and $\mu$ are the constant values for different modulation schemes detailed in [31] and $\Gamma(.,$.$) denotes the incomplete Gamma function.$

Substituting (18) into (26), the ABER for the concerned system under binary modulation can be expressed as: 


$$
\begin{aligned}
& P_{b}=\frac{\Psi^{k_{q}+m_{q}}-\left(k_{q}+m_{q}\right) / 2\left(\frac{m_{P} \bar{\gamma}_{I}}{m_{I} \bar{\gamma}_{P}}\right)^{\left(k_{q}+m_{q}\right) / 2}}{2 \Gamma(\psi) \Gamma\left(k_{q}\right) \Gamma\left(m_{q}\right) \Gamma\left(m_{I} N_{I}\right) \Gamma\left(m_{P} N_{P}\right)\left(\bar{\gamma}_{R} / D_{R}\right)^{\left(k_{q}+m_{q}\right) / 2}} \int_{0}^{\infty} \gamma^{\left(\left(k_{q}+m_{q}\right) / 2-1\right)} \Gamma(\psi, \mu x) \\
& \times G_{1,3}^{3,1}\left(\frac{\Psi^{2} \bar{\gamma}_{I} m_{P} D_{R} \gamma}{m_{I} \bar{\gamma}_{R} \bar{\gamma}_{P} \xi} \mid \begin{array}{c}
\left(k_{q}+m_{q}\right) / 2+m_{I} N_{I} \\
m_{P} N_{P}-\left(\left(k_{q}+m_{q}\right) / 2\right),\left(k_{q}+m_{q}\right) / 2,\left(m_{q}-k_{q}\right) / 2
\end{array}\right) d \gamma,
\end{aligned}
$$

By converting the $\Gamma(\psi, \mu x)$ to the Meijer-G function using the identity detailed in ([30], Equation $(8.4 .16(2)))$, then (27) can be expressed as:

$$
\begin{aligned}
P_{b}= & \frac{\Psi^{k_{q}+m_{q}} \xi^{-\left(k_{q}+m_{q}\right) / 2}\left(\frac{m_{P} \bar{\gamma}_{I}}{m_{I} \bar{\gamma}_{P}}\right)^{\left(k_{q}+m_{q}\right) / 2}}{2 \Gamma(\psi) \Gamma\left(k_{q}\right) \Gamma\left(m_{q}\right) \Gamma\left(m_{I} N_{I}\right) \Gamma\left(m_{P} N_{P}\right)\left(\bar{\gamma}_{R} / D_{R}\right)^{\left(k_{q}+m_{q}\right) / 2}} \int_{0}^{\infty} \gamma^{\left(\left(k_{q}+m_{q}\right) / 2-1\right)} G_{1,2}^{2,0}\left(\mu x \mid \begin{array}{c}
1 \\
0, \psi
\end{array}\right) \\
& \times G_{1,3}^{3,1}\left(\frac{\Psi^{2} \bar{\gamma}_{I} m_{P} D_{R} \gamma}{m_{I} \bar{\gamma}_{R} \bar{\gamma}_{P} \xi} \mid \begin{array}{c}
\left(k_{q}+m_{q}\right) / 2+m_{I} N_{I} \\
m_{P} N_{P}-\left(\left(k_{q}+m_{q}\right) / 2\right),\left(k_{q}+m_{q}\right) / 2,\left(m_{q}-k_{q}\right) / 2
\end{array}\right) d \gamma,
\end{aligned}
$$

Using the integral identity given in [24], Equations (21) and (28) can be solved as:

$$
\begin{gathered}
P_{b}=\frac{\Psi^{k_{q}+m_{q} \xi}-\left(k_{q}+m_{q}\right) / 2\left(\frac{m_{P} \bar{\gamma}_{I}}{m_{I} \bar{\gamma}_{P}}\right)^{\left(k_{q}+m_{q}\right) / 2}}{2 \Gamma(\psi) \Gamma\left(k_{q}\right) \Gamma\left(m_{q}\right) \Gamma\left(m_{I} N_{I}\right) \Gamma\left(m_{P} N_{P}\right)\left(\bar{\gamma}_{R} / D_{R}\right)^{\left(k_{q}+m_{q}\right) / 2}} \\
\times G_{3,4}^{3,3}\left(\frac{\Psi^{2} \bar{\gamma}_{I} m_{P} D_{R} \gamma}{\mu m_{I} \bar{\gamma}_{R} \bar{\gamma}_{P} \xi} \mid \begin{array}{c}
\left(k_{q}+m_{q}\right) / 2+m_{I} N_{I}, 1-\left(\left(k_{q}+m_{q}\right) / 2\right),-\left(\left(k_{q}+m_{q}\right) / 2\right)-\psi \\
m_{P} N_{P}-\left(\frac{\left(k_{q}+m_{q}\right)}{2}\right),\left(k_{q}+m_{q}\right) / 2,\left(m_{q}-k_{q}\right) / 2,-\left(\left(k_{q}+m_{q}\right) / 2\right)
\end{array}\right) .
\end{gathered}
$$

\subsubsection{For M-Ary Coherent Modulation}

The average BER for the concerned system under the $M$-ary coherent modulation can be defined as [32,33]:

$$
P_{b}=\varphi \sum_{i=1}^{t} \int_{0}^{\infty} f_{\gamma}(\gamma) Q\left(\sqrt{\Xi_{i} \gamma}\right) d \gamma,
$$

where $\varphi, t$ and $\Xi_{i}$ are the parameters that determine the type of $M$-ary coherent modulation, and they are defined in $[7,33]$ for M-PSK and M-QAM.

Invoking (18) into (30), the ABER under this modulation can be expressed as:

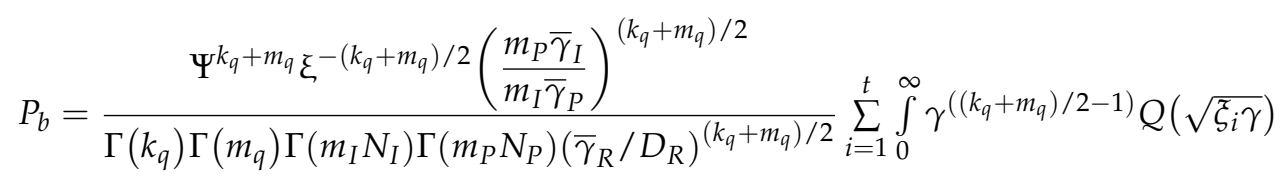

$$
\begin{aligned}
& \times G_{1,3}^{3,1}\left(\frac{\Psi^{2} \bar{\gamma}_{I} m_{P} D_{R} \gamma}{m_{I} \bar{\gamma}_{R} \bar{\gamma}_{P} \xi} \mid \begin{array}{c}
\left(k_{q}+m_{q}\right) / 2+m_{I} N_{I} \\
m_{P} N_{P}-\left(\left(k_{q}+m_{q}\right) / 2\right),\left(k_{q}+m_{q}\right) / 2,\left(m_{q}-k_{q}\right) / 2
\end{array}\right) d \gamma, \\
& \text { If } Q\left(\sqrt{\Xi_{i} \gamma}\right)=\frac{1}{2} \operatorname{erf} c\left(\sqrt{\frac{\Xi_{i}}{2} \gamma}\right) \text { and is further converted to the Meijer-G function using }
\end{aligned}
$$




$$
\begin{gathered}
P_{b}=\frac{\Psi^{k_{q}+m_{q}} \xi^{-\left(k_{q}+m_{q}\right) / 2}\left(\frac{m_{P} \bar{\gamma}_{I}}{m_{I} \bar{\gamma}_{P}}\right)^{\left(k_{q}+m_{q}\right) / 2}}{2 \sqrt{\pi} \Gamma\left(k_{q}\right) \Gamma\left(m_{q}\right) \Gamma\left(m_{I} N_{I}\right) \Gamma\left(m_{P} N_{P}\right)\left(\bar{\gamma}_{R} / D_{R}\right)^{\left(k_{q}+m_{q}\right) / 2}} \sum_{i=1}^{t} \int_{0}^{\infty} \gamma^{\left(\left(k_{q}+m_{q}\right) / 2-1\right)} \\
\times G_{1,2}^{2,0}\left(\frac{\Xi_{i}}{2} x \mid \begin{array}{c}
1 \\
0,1 / 2
\end{array}\right) G_{1,3}^{3,1}\left(\frac{\Psi^{2} \bar{\gamma}_{I} m_{P} D_{R} \gamma}{m_{I} \bar{\gamma}_{R} \bar{\gamma}_{P} \xi} \mid \begin{array}{c}
\left(k_{q}+m_{q}\right) / 2+m_{I} N_{I} \\
m_{P} N_{P}-\left(\left(k_{q}+m_{q}\right) / 2\right),\left(k_{q}+m_{q}\right) / 2,\left(m_{q}-k_{q}\right) / 2
\end{array}\right) d \gamma,
\end{gathered}
$$

By applying the integral identity detailed in [24], Equations (21) and (32) can be solved as:

$$
\begin{aligned}
& P_{b}=\frac{\Psi^{k_{q}+m_{q}} \xi^{-\left(k_{q}+m_{q}\right) / 2}}{2 \sqrt{\pi} \Gamma\left(k_{q}\right) \Gamma\left(m_{q}\right) \Gamma\left(m_{I} N_{I}\right) \Gamma\left(m_{P} N_{P}\right)\left(\bar{\gamma}_{R} / D_{R}\right)^{\left(k_{q}+m_{q}\right) / 2}} \sum_{i=1}^{t}\left(\frac{2 m_{P} \bar{\gamma}_{I}}{\Xi_{i} m_{I} \bar{\gamma}_{P}}\right)^{\left(k_{q}+m_{q}\right) / 2} \\
& \times G_{3,4}^{3,3}\left(\frac{2 \Psi^{2} \bar{\gamma}_{I} m_{P} D_{R} \gamma}{\Xi_{i} m_{I} \bar{\gamma}_{R} \bar{\gamma}_{P} \xi} \mid \begin{array}{c}
\left(k_{q}+m_{q}\right) / 2+m_{I} N_{I} \\
m_{P} N_{P}-\left(\left(k_{q}+m_{q}\right) / 2\right),\left(k_{q}+m_{q}\right) / 2,\left(m_{q}-k_{q}\right) / 2,-\left(\left(k_{q}+m_{q}\right) / 2\right)
\end{array}\right) .
\end{aligned}
$$

\section{Numerical Results and Discussions}

In this section, the numerical results are presented to evaluate the impact of various system and channel parameters on the performance of RIS in an interference-limited wireless powered communication network. It can be observed that the Monte-Carlo simulation results are perfectly matched with the analytical results, which indicate the correctness of the derived closed-form expressions. Unless otherwise stated, the system and channel parameters are set to be as follows: $R=2, P_{I}=15 \mathrm{~dB}, N_{p}=N_{I}=2, m_{p}=m_{I}=2$, $\bar{\gamma}_{p}=\bar{\gamma}_{I}=\bar{\gamma}_{R}=5 \mathrm{~dB}, \zeta=2.5, \rho=0.6, \beta=0.8, A=1, d_{p}=2 \mathrm{~m}, d_{I}=10 \mathrm{~m}$ and $d_{A R}=d_{R D}=2 \mathrm{~m}$.

In Figure 2, the impact of the number of reflecting elements in the RIS on the system outage probability is demonstrated. It can be observed from the results that the increase in number of reflecting elements in the RIS significantly improves the system outage performance with large values of $N$ offering better performance. Further, it is determined from the results that the proposed system with RIS outperforms the conventional system without RIS.

The effect of number of interferers $N_{I}$ and the number of antennas $N_{p}$ on the system outage probability is presented in Figure 3 . The results show that the system performance significantly deteriorates with an increase in $N_{I}$. However, the system offers better performance as the number of antennas $N_{p}$ at the PB increases. This is because the increase in $N_{p}$ increases the received power at the system destination.

Moreover, the performance of the system under the variation of interferers transmission power $P_{I}$ and the fading parameters $m_{p}$ and $m_{I}$ on the PB and interferer links, respectively, is depicted in Figure 4. It can be deduced that the increase in the transmit power $P_{I}$ of interferers leads to degradation of the system outage performance. However, the results indicate that the increment in the fading parameters on the PB and interferer links enhances the system outage performance.

The throughput performance of the system under the variation of $N_{I}$ is presented in Figure 5. The results show that the system performance drastically degrades with an increase in the number of interferers at the system destination. 


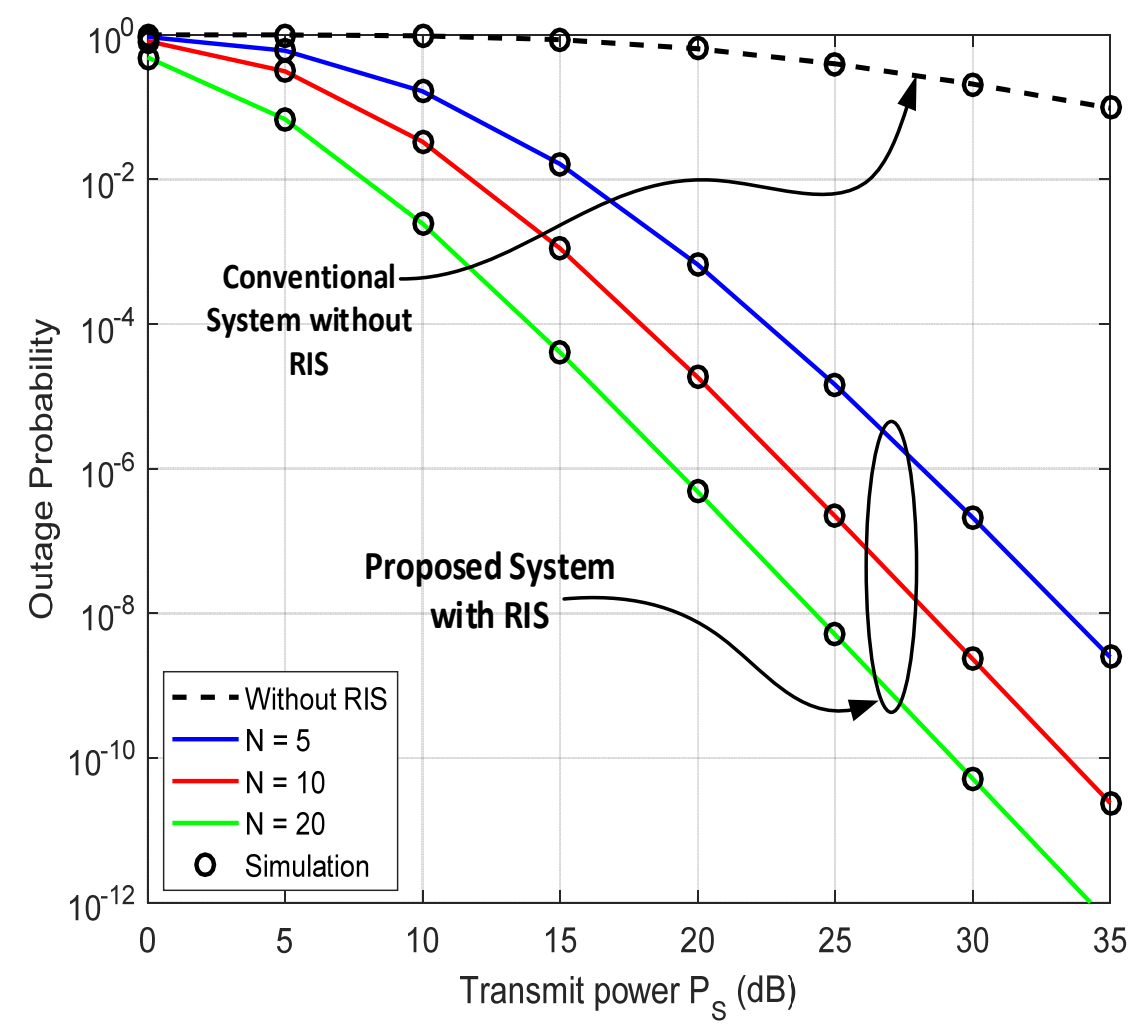

Figure 2. Outage performance of the concerned system for various values of $N$.

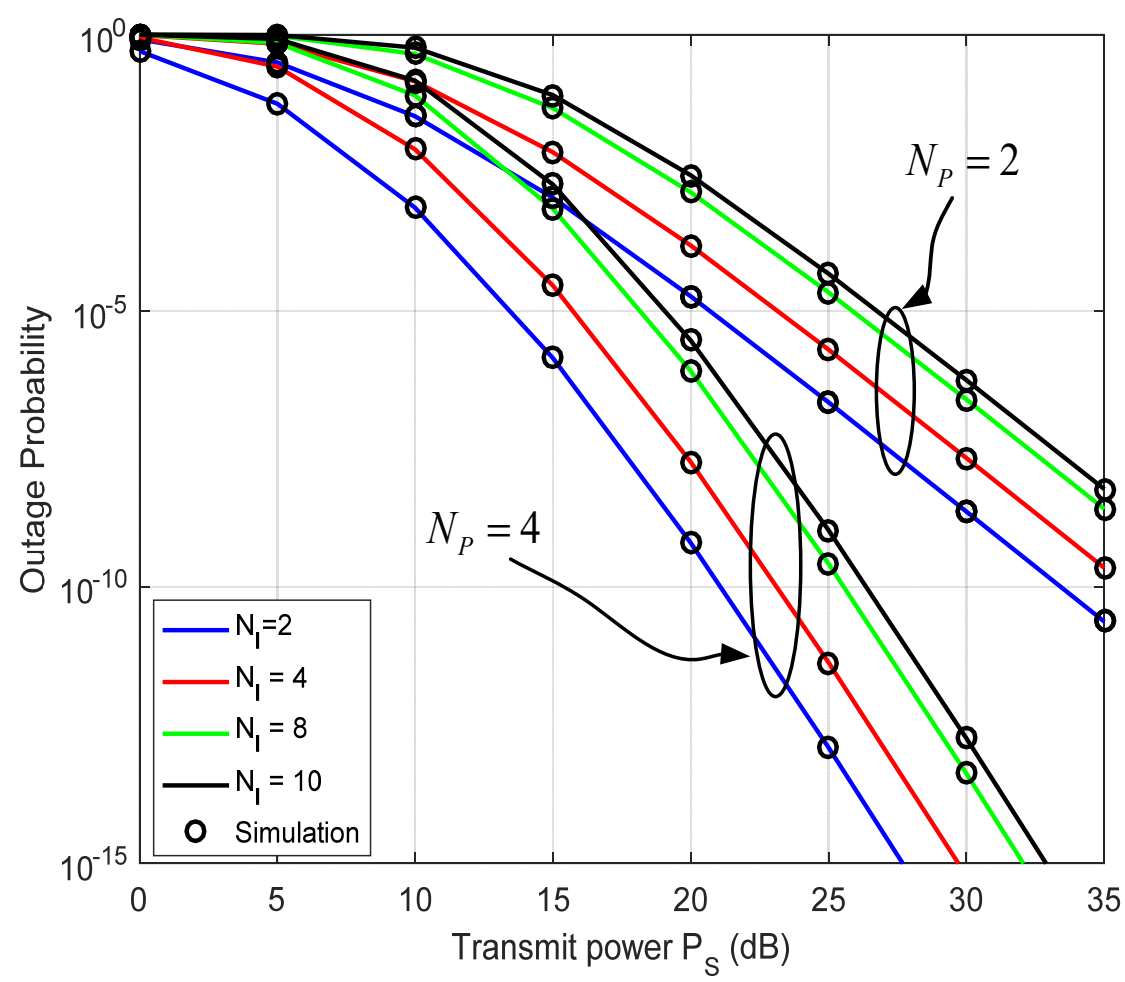

Figure 3. Outage probability performance at various values of $N_{I}$ and $N_{p}$ at $N=10$. 


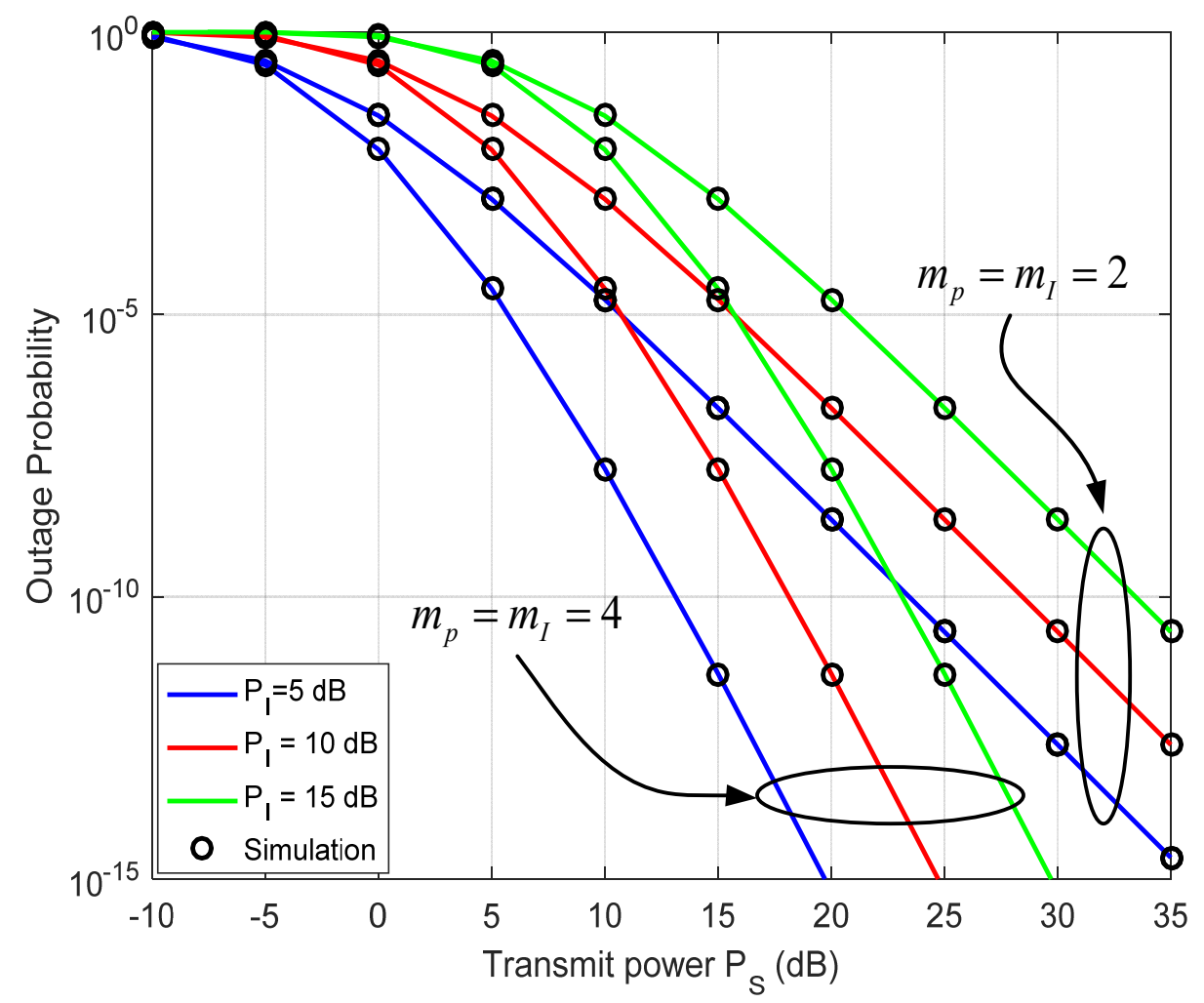

Figure 4. Impact of $P_{I}$ on the system outage probability performance at various values of $m_{I}$ and $m_{p}$ at $N=10$.

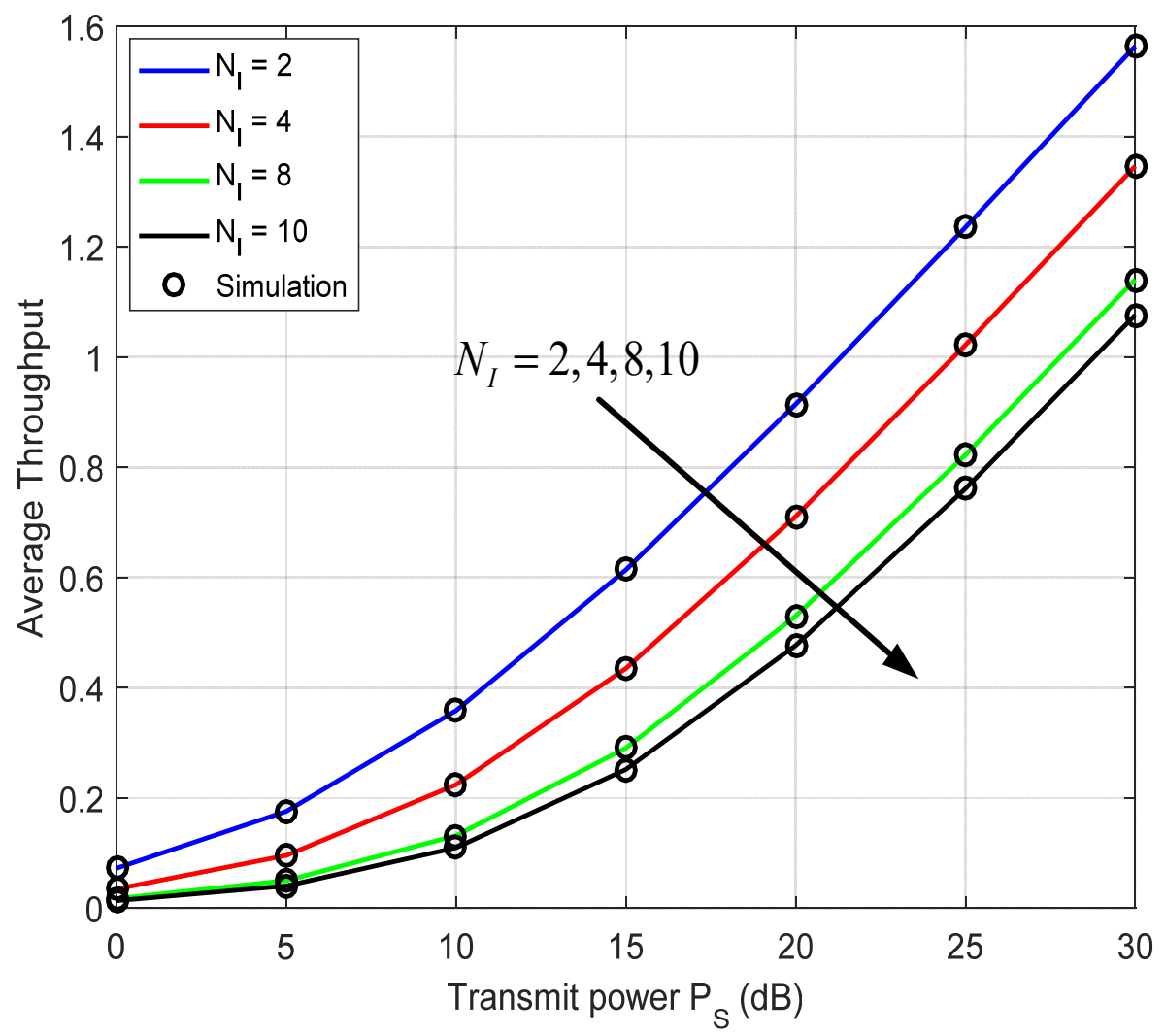

Figure 5. Effect of the number of interferers on the average throughput when $N=10$. 
The impact of number of antennas $N_{p}$ at the PB on the system throughput is demonstrated in Figure 6. The results illustrate that the system average throughput increases with an increase in $N_{p}$. Further, it can be observed from the results that the system throughput improves with an increase in energy harvesting time $\rho$. However, maximum throughput is achieved when $\rho$ reaches its optimum value. This is due to the fact that more time is allocated to harvesting energy from the $\mathrm{PB}$, which leads to an increase in the system throughput.

Similarly, the impact of number of reflecting elements in RIS on the system throughput is depicted in Figure 7. The results show that the proposed system with an RIS achieves higher throughput compared to a conventional system without RIS as the $N$ increases.

In Figure 8, the impact of the number of reflecting elements in an RIS and the fading parameter on the PB and interferer links on the system ABER of the system under the BPSK binary modulation scheme is presented. It can be seen that the increase in the number of $N$ significantly improved the ABER, with higher value offering the system better performance. The results also indicate that the increase in the system fading parameters on both the PB and interferer links leads to better system performance. Additionally, it can be seen that the conventional system without RIS offers poor performance when compared with the proposed system with RIS.

The effects of the number of interferers on the system ABER under the M-QAM coherent modulation system is presented in Figure 9. It can be observed that the system ABER degraded as the number of interferers increased at the destination. However, as the number modulation order $M$ decreased, the better the system ABER performance.

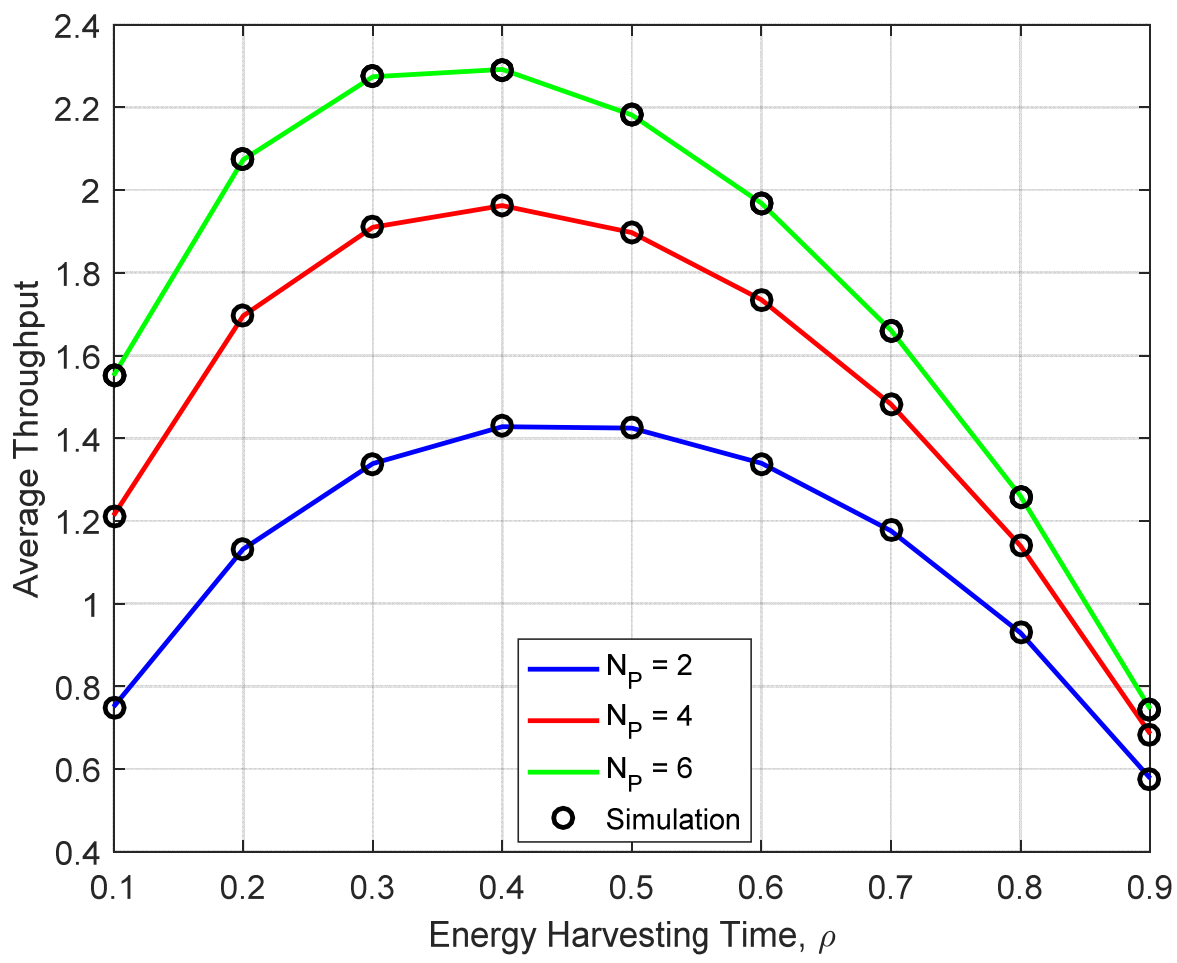

Figure 6. Average throughput vs. energy harvesting time $\rho$ under different values of $N_{p}$ at $N=10$. 


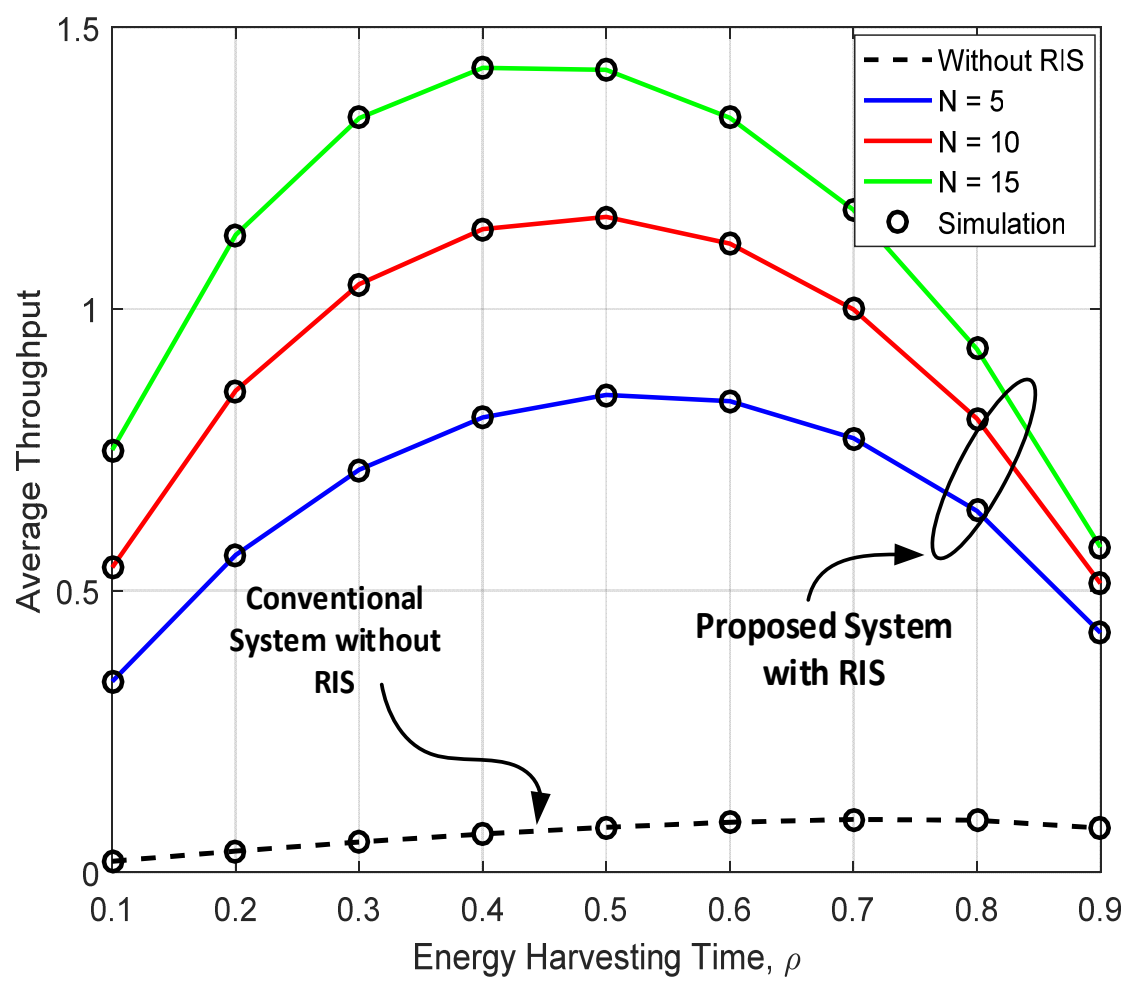

Figure 7. Impact of number of reflecting elements on the average throughput under different system scenarios.

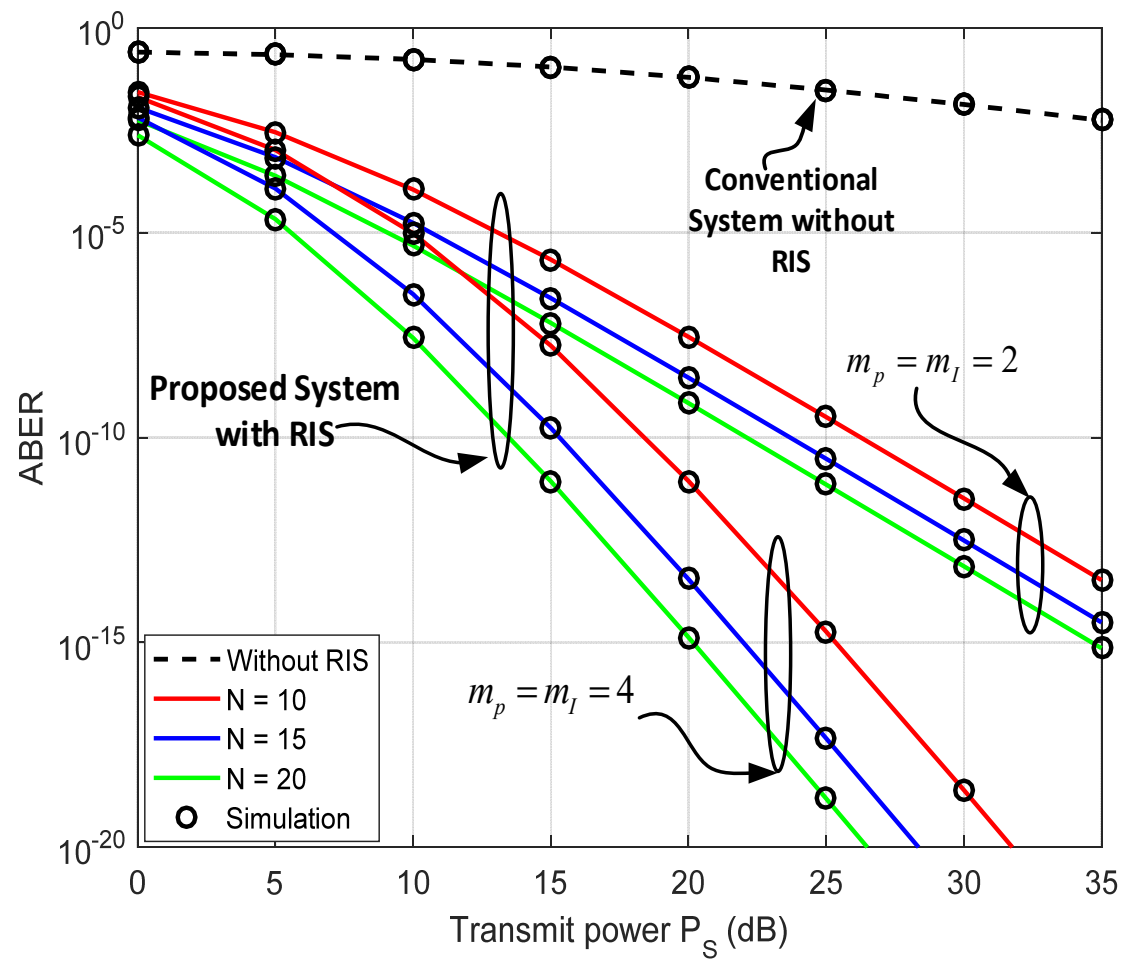

Figure 8. Effect of number of reflecting elements in the RIS and fading parameters on the system average BER under binary modulation scheme. 


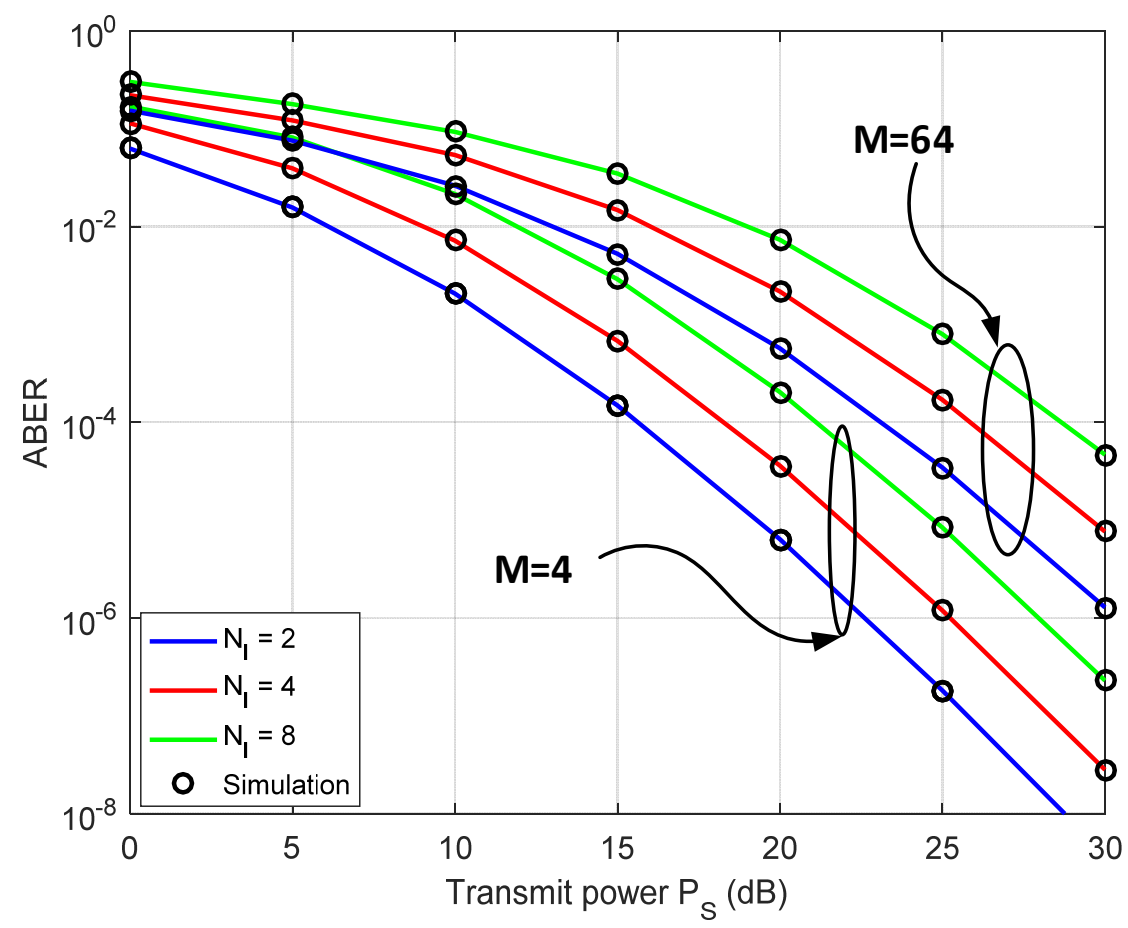

Figure 9. Effect of number of reflecting elements in the RIS and the modulation order on the system average BER under M-QAM coherent modulation scheme.

\section{Conclusions}

In this paper, the performance of the RIS in a wireless powered limited interference communication network is analysed. The exact closed-form expressions of the system outage probability, average throughput and average bit error rate are derived. The results depicted that the simulation results perfectly agreed with the analytical results. The results also show that the system performance significantly improves with an increase in the number of RIS reflecting elements, number of antennas at the PB and fading parameter on the $\mathrm{PB}$ and interferer links. Moreover, the system performance deteriorates as the number of interferes and $P_{I}$ increase. Further, the system average throughput increases with the increase in energy harvesting time. The results also demonstrated that the proposed system with the RIS offers better performance compared with the conventional dual-hop system without the RIS. The furture research interest of this work can be directed towards the impact of RIS surafcae area on the performance of system. This is owing to the fact that the smaller RIS reflecting elements offer better robustness, but at higher design complexity. However, RIS with large element structures aid better reflection, but at the expense of higher power consumption. Moreover, the system can be made multi-user and the effect of hardware impairment with co-channel interference can be studied.

Author Contributions: Conceptualization, K.O. and P.O.; methodology, K.O.; software, K.O. and O.O.; validation, K.O. P.O. and O.O.; formal analysis, K.O.; writing-original draft preparation, K.O.; writing-review and editing, K.O. P.O. and O.O.; supervision, P.O. All authors have read and agreed to the published version of the manuscript.

Funding: This research received no external funding.

Institutional Review Board Statement: Not applicable.

Informed Consent Statement: Not applicable.

Data Availability Statement: No new data were created or analyzed in this study. Data sharing is not applicable to this article.

Conflicts of Interest: The authors declare no conflict of interest. 


\section{References}

1. Basar, E.; Di Renzo, M.; De Rosny, J.; Debbah, M.; Alouini, M.-S.; Zhang, R. Wireless communications through reconfigurable intelligent surfaces. IEEE Access 2019, 7, 116753-116773. [CrossRef]

2. Dixit, D.; Joshi, K.C.; Sharma, S. Performance of Intelligent Reconfigurable Surface-Based Wireless Communications Using QAM Signaling. arXiv 2020, arXiv:2010.00519.

3. Basar, E. Transmission through large intelligent surfaces: A new frontier in wireless communications. In Proceedings of the 2019 European Conference on Networks and Communications (EuCNC), Valencia, Spain, 18-21 June 2019; pp. 112-117.

4. Odeyemi, K.O.; Owolawi, P.A.; Olakanmi, O.O. Performance Analysis of Reconfigurable Intelligent Surface Assisted Underwater Optical Communication System. Prog. Electromagn. Res. 2020, 98, 101-111. [CrossRef]

5. Wu, Q.; Zhang, R. Towards smart and reconfigurable environment: Intelligent reflecting surface aided wireless network. IEEE Commun. Mag. 2019, 58, 106-112. [CrossRef]

6. Samuh, M.H.; Salhab, A.M. Performance analysis of reconfigurable intelligent surfaces over Nakagami-m fading channels. arXiv 2020, arXiv:2010.07841.

7. Badarneh, O.S.; Da Costa, D.B.; Nardelli, P.H.J. Wireless-powered communication networks with random mobility. IEEE Access 2019, 7, 166476-166492. [CrossRef]

8. Badarneh, O.S. A comprehensive analysis of the achievable throughput in interference-limited wireless-powered networks with nonlinear energy harvester. Trans. Emerg. Telecommun. Technol. 2020, 31, e4141. [CrossRef]

9. Messadi, O.; Sali, A.; Khodamoradi, V.; Salah, A.A.; Pan, G.; Hashim, S.J.; Noordin, N.K. Optimal Relay Selection Scheme with Multiantenna Power Beacon for Wireless-Powered Cooperation Communication Networks. Sensors 2021, $21,147$. [CrossRef] [PubMed]

10. Badarneh, O.S.; Almehmadi, F.S.; Ansari, I.S.; Yang, X. Wireless energy harvesting in cooperative decode-and-forward relaying networks over mixed generalized $\eta-\mu$ and $\kappa-\mu$ fading channels. Trans. Emerg. Telecommun. Technol. 2018, 29, e3262. [CrossRef]

11. Nasir, A.A.; Zhou, X.; Durrani, S.; Kennedy, R.A. Relaying protocols for wireless energy harvesting and information processing. IEEE Trans. Wirel. Commun. 2013, 12, 3622-3636. [CrossRef]

12. Yang, L.; Meng, F.; Zhang, J.; Hasna, M.O.; Di Renzo, M. On the performance of RIS-assisted dual-hop UAV communication systems. IEEE Trans. Veh. Technol. 2020, 69, 10385-10390. [CrossRef]

13. Odeyemi, K.O.; Owolawi, P.A.; Olakanmi, O.O. On the Performance of Reconfigurable Intelligent Surface Aided Power Line Communication System under Different Relay Transmission Protocols. Prog. Electromagn. Res. C 2021, 111, 119-133. [CrossRef]

14. Salhab, A.M. Performance Analysis of RIS-Assisted Source Mixed RF/FSO Relay Networks. arXiv 2020, arXiv:2011.05612.

15. Odeyemi, K.O.; Owolawi, P.A.; Olakanmi, O.O. Reconfigurable intelligent surface assisted mobile network with randomly moving user over Fisher-Snedecor fading channel. Phys. Commun. 2020, 43, 101186. [CrossRef]

16. Makarfi, A.U.; Rabie, K.M.; Kaiwartya, O.; Badarneh, O.S.; Li, X.; Kharel, R. Reconfigurable intelligent surface enabled IoT networks in generalized fading channels. In Proceedings of the ICC 2020-2020 IEEE International Conference on Communications (ICC), Virtual Conference, Dublin, Ireland, 7-11 June 2020; pp. 1-6.

17. Canbilen, A.E.; Basar, E.; Ikki, S.S. Reconfigurable intelligent surface-assisted space shift keying. IEEE Wirel. Commun. Lett. 2020, 9, 1495-1499. [CrossRef]

18. Yue, X.; Liu, Y. Performance analysis of intelligent reflecting surface assisted NOMA networks. arXiv 2020, arXiv:2002.09907.

19. Yang, L.; Guo, W.; da Costa, D.B.; Alouini, M.-S. Free-Space Optical Communication with Reconfigurable Intelligent Surfaces. arXiv 2020, arXiv:2012.00547.

20. Makarfi, A.U.; Rabie, K.M.; Kaiwartya, O.; Li, X.; Kharel, R. Physical layer security in vehicular networks with reconfigurable intelligent surfaces. In Proceedings of the 2020 IEEE 91st Vehicular Technology Conference (VTC2020-Spring), Antwerp, Belgium, 25-28 May 2020; pp. 1-6.

21. Ai, Y.; Kong, L.; Cheffena, M.; Chatzinotas, S.; Ottersten, B. Secure Vehicular Communications through Reconfigurable Intelligent Surfaces. arXiv 2020, arXiv:2011.14899.

22. Yang, L.; Yang, Y.; da Costa, D.B.; Trigui, I. Performance Analysis of an Interference-Limited RIS-Aided Network. arXiv 2020, arXiv:2011.07479.

23. Yang, L.; Meng, F.; Wu, Q.; da Costa, D.B.; Alouini, M.-S. Accurate closed-form approximations to channel distributions of RIS-aided wireless systems. IEEE Wirel. Commun. Lett. 2020, 9, 1985-1989. [CrossRef]

24. Adamchik, V.; Marichev, O. The algorithm for calculating integrals of hypergeometric type functions and its realization in REDUCE system. In Proceedings of the International Symposium on Symbolic and Algebraic Computation, Tokyo, Japan, 20-24 August 1990; pp. 212-224.

25. Yang, L.-L.; Chen, H.-H. Error probability of digital communications using relay diversity over Nakagami-m fading channels. IEEE Trans. Wirel. Commun. 2008, 7, 1806-1811. [CrossRef]

26. Nguyen, H.-N.; Nguyen, N.-L.; Nguyen, N.-T.; Le, A.-T.; Ha, N.-D.X.; Do, D.-T.; Voznak, M. Reliable and Secure Transmission in Multiple Antennas Hybrid Satellite-Terrestrial Cognitive Networks Relying on NOMA. IEEE Access 2020, 8 , 215044-215056. [CrossRef]

27. Gradshteyn, I.; Ryzhik, I.; Romer, R.H. Tables of Integrals, Series, and Products; American Association of Physics Teachers: College Park, MD, USA, 1988. 
28. Zedini, E.; Ansari, I.S.; Alouini, M.-S. Performance analysis of mixed Nakagami- $m$ and Gamma-Gamma dual-hop FSO transmission systems. IEEE Photonics J. 2014, 7, 1-20. [CrossRef]

29. Bhutto, Z.; Yoon, W. Dual-Hop Cooperative Relaying with Beamforming Under Adaptive Transmission in $\kappa-\mu$ Shadowed Fading Environments. Electronics 2019, 8, 658. [CrossRef]

30. Prudnikov, A.; Brychkov, Y.A.; Marichev, O. Integrals and Series Volume 3: More Special Functions; Taylor and Francis: Oxford, UK, 2003.

31. Simon, M.K.; Alouini, M.-S. Digital Communication over Fading Channels; John Wiley \& Sons: Hoboken, NJ, USA, $2005 ;$ Volume 95.

32. Lu, J.; Letaief, K.B.; Chuang, J.-I.; Liou, M.L. M-PSK and M-QAM BER computation using signal-space concepts. IEEE Trans. Commun. 1999, 47, 181-184.

33. Badarneh, O.S.; Sofotasios, P.C.; Muhaidat, S.; Cotton, S.L.; Da Costa, D.B. Product and Ratio of Product of Fisher-Snedecor $\mathcal{F}$ Variates and Their Applications to Performance Evaluations of Wireless Communication Systems. IEEE Access 2020, 8 , 215267-215286. [CrossRef] 